\title{
EL TESTAMENTO Y OTROS DOCUMENTOS SOBRE DANIELE DA VOLTERRA*
}

\author{
POR \\ GonZALO Redín \\ Museo Nacional del Prado
}

Daniele da Volterra es más conocido en España por pintar los paños que cubren algunos de los desnudos del Juicio final de Miguel Ángel, que por su obra, que le define como el más fiel heredero de su maestro. Sin embargo, su influencia en el arte español a través de Gaspar Becerra, discípulo suyo en Roma ${ }^{1}$, condicionó el desarrollo de la escultura en buena parte de nuestro país en la segunda mitad del siglo XVI. Publicamos y comentamos aquí su testamento inédito, localizado en el Archivio di Stato di Roma entre los volúmenes del notario Thomassino ${ }^{2}$, que se encargó del inventario de sus bienes ${ }^{3}$, y aportamos noticias relativas a su herencia y a sus discípulos directos, Michele Alberti, Feliciano de San Vito y Biagio Betti, e indirectos, como Jacopo Rocchetti.

Palabras clave: Daniele Ricciarelli da Volterra; Testamento; Michele Alberti; Feliciano de San Vito; Biagio Betti; Jacopo Rocchetti.

THE TESTAMENT AND OTHER DOCUMENTS CONCERNING DANIELE DA VOLTERRA

Daniele da Volterra is better known in Spain for painting the drapery that covers some of the nudes in Michelangelo's The Last Judgment than for his own work, which defined him as his master's most loyal successor. Nonetheless, Daniele's influence on Spanish art through Gaspar Becerra, a disciple of his

\footnotetext{
* Quiero agradecer a Sandro Corradini, Fernando Bilancia y Micaela Antoniucci su colaboración en la transcripción y lectura de algunos documentos.

${ }^{1}$ Redín, G., Pedro Rubiales, Gaspar Becerra y los pintores españoles en Roma. 1527-1600, Tesis Doctoral, Universidad Autónoma de Madrid, 2005. Redín, G., Pedro Rubiales, Gaspar Becerra y los pintores españoles en Roma, Madrid, CSIC, 2007. Para Longhi, los frescos de Becerra en el Palacio de El Pardo son lo más similar al arte de Daniele que pueda encontrarse. Longhi, P., "Due pannelli di Daniele da Volterra”, Paragone, 179, 1964, p. 36.

2 Citado, sin su transcripción en Redín, 2005, p. 179, n. 693. Redín, 2007, p. 166. ASR (Archivio di Stato di Roma), CNC (Collegio Notai Capitolini), 1772, Thomassinus, 4 de abril 1566, f. 284 r y v.

${ }^{3}$ Publicado por Gasparoni parcialmente sin señalar su ubicación. Hay dos versiones del inventario, ambas en el Archivio di Stato di Roma. Una en Miscellanea Corvisieri, busta 1, fascicolo 50 y otra entre los volúmenes del notario Thomassinus, CNC 1772, f. 287 r y 307-308. GASPARONI, B., "La casa di Michelangelo Buonarroti”, Il Buonarroti, 1, 1866, pp. 158-164, 177-180, 204-207. CorBo, A. M., "Documenti romani su Michelangelo", Commentari, 16, 1965, pp. 98-151 (107).
} 
in Rome, determined to a large extent the development of sculpture in this country in the second half of the 16th century. This article makes known and discusses Daniele's previously unpublished last will and testament, located in the Archivio di Stato di Roma among the volumes by the notary Thomassino, who attended to the inventory of his possessions. It also provides new details on Daniele's estate and on his direct disciples Michele Alberti, Feliciano de San Vito, and Biagio Betti along with his indirect ones such as Jacopo Rocchetti.

Key words: Daniele Ricciarelli da Volterra; Testament; Heir; Michele Alberti; Feliciano de San Vito; Biagio Betti; Jacopo Rocchetti.

Según refiere Vasari, Daniele Ricciarelli da Volterra murió el 4 de abril de 1566 de un "catarro crudele", agravado por los disgustos que le dio la complicada fundición del caballo de la colosal estatua ecuestre de Enrique II de Francia ${ }^{4}$. El aretino da cuenta del fallecimiento a Vincenzo Borghini en una carta en la que detalla que ocurrió "... dopo quattro giorni di passione d'animo, che '1 suo cavallo non venne bene la prima volta e l'à auto a rigettare. Et ancora è nella fossa sotterrato, talchè ha messo sotto terra "l maestro" . Esta noticia no es exagerada, puesto que Daniele dictó su testamento, trágicamente, en la misma fragua donde se encontraba la obra, cuya finalización, al parecer, le costó la vida ese mismo día. Apremiado por su estado de salud, Daniele hizo testamento en el domicilio de cierto Attilio Ceci, que estaba situado junto a su casa. Algunas fuentes indican que la estatua del caballo de Enrique II se fundió en el taller del "Capitan Martio Ceci", ubicado en el Quirinale, junto a la iglesia de San Gerolamo, en Montecavallo ${ }^{6}$. Seguramente Attilio era pariente de Martio Ceci, o bien se trata de la misma persona, cuyo nombre, por su similitud, pudo ser confundido ${ }^{7}$, pues su domicilio estaba precisamente junto a la casa de Ricciarelli, lo que cuadra con la "stanza molto a proposito" que, según Vasari, Daniele tenía en Montecavallo para hacer la estatua ${ }^{8}$.

Diez años antes, el 8 de agosto de 1556 cierto Fabio de Bentornato alquiló a Daniele da Volterra por 18 escudos anuales una casa en el Rione Trevi. La vivienda estaba situada junto al domicilio de cierto Pietro de Varis y al del propio arrendador, que creó un censo de 50 escudos sobre la habitación. Daniele pagó la mitad del censo ese mismo día, comprometiéndose a pagar la otra mitad en septiembre, lo que no hizo, por lo que el 2 de octubre se anuló la compra ${ }^{9}$. El 8 de octubre de 1556, cierto "Ludovicus qd Johannes de Fliano, cirurgicus" vendía una casa de su propiedad que se encontraba "in regione trivii et apud capuccinos cui ab uno sunt bona danielis de ricciarellis ab alio bono viro Fabii de Bentornatis" ${ }^{\prime 10}$. Es decir, junto a la iglesia de los capu-

\footnotetext{
${ }^{4}$ VASARI, G., Le vite de' più eccellenti pittori, scultori et architettori: con i ritratti loro et con l'aggiunta delle Vite de' vivi \& de' morti dall'anno 1550 insino al 1567, Florencia 1568, V, p. 549.

${ }^{5}$ La carta se fecha el 14 de abril. Frey, K., Carteggio del Vasari, II, Munich, 1930, pp. 128-129.

${ }^{6}$ Llamada antes San Salvatore de' Cornelii, hasta que los "Gesuati" la dedicaron a su patrón, fue destruida en tiempos de Paolo V, y se ubicaba en lo que es hoy el jardín del palacio Rospigliosi, en Montecavallo. ArmeLLINI, M., Le chiese di Roma: dal secolo IV al XIX, Roma, 1942, p. 229. Así lo indica Ferrucci, que habla de la iglesia de "San Geronimo", informado en 1600 por Michele Alberti, uno de los discípulos de Daniele, y lo corrobora en 1583 Jacomo Corte, agente del duque de Saboya. Ferrucci, G., L'antichità di Roma di Andrea Fulvio... con le aggiuntioni e annotationi di Girolamo Ferrucci, Roma, 1600, pp. 320-21. CORTE en Documenti inediti per servire alla storia dei Musei d'Italia, II, Florencia y Roma, 1879, p. 401. Böstrom, A., "Daniele da Volterra and the Equestrian Monument to Henry II of France", The Burlington Magazine, 137, 1995, pp. 809-820 (815).

7 Además, Corte y Ferrucci escribieron, respectivamente, 17 y 34 años después de la fusión del caballo.

8 VASARI, 1568, VI, p. 548.

9 El documento está anulado con una x. ASR. CNC. 31, Notario Amadeis, 8 agosto 1556, ff. 342 r y v.

10 ASR. CNC. 31, Amadeis, 8 de octubre de 1556, ff. 388 r y v. También este documento está tachado.
} 
chinos $^{11}$, y ubicada entre una vivienda de Bentornato y otra propiedad de Daniele. Estas casas no son citadas en el testamento ni en el inventario, pero sí es seguro que en 1566 vivía en el Rione Monti, en Montecavallo, muy cerca de la iglesia de San Gerolamo y junto a la casa de Attilio Ceci.

Sin embargo, pese a la cercanía de estos templos, Daniele ordenó ser sepultado en la nueva Basílica de Santa Maria degli Angeli, último gran proyecto de Miguel Ángel, iniciado en 1562, para transformar en iglesia la parte central de las antiguas Termas de Diocleciano. Dada su íntima relación con Buonarroti ${ }^{12}$, no sorprende que Ricciarelli quisiera ser enterrado en la nueva basílica, habida cuenta de que el siciliano Jacopo del Duca, también estrecho colaborador del de Caprese, había escrito en marzo de 1565 al sobrino de Miguel Ángel, Leonardo Buonarroti, contándole que iba a realizar, a sus expensas y siguiendo un diseño del maestro, un tabernáculo destinado a la nueva basílica para honrar su memoria ${ }^{13}$. Así, Jacopo se resarcía del sepulcro de Miguel Ángel que, bajo diseño de Daniele, él y Jacomo Rocchetti no pudieron acometer cuando se decidió que el genio fuera enterrado en Florencia ${ }^{14}$. Daniele fue la primera persona inhumada en Santa Maria degli Angeli después de Antonio del Duca, tío de Jacopo del Duca, amigo de Buonarroti y promulgador del culto angélico que dio origen a la basílica ${ }^{15}$. En la nueva iglesia trabajaría, en lo sucesivo, una "cerchia" de artistas directamente relacionados con los Del Duca, Ricciarelli y Buonarroti. En el testamento de Antonio del Duca, redactado en octubre de 1564, aparecen como testigos un discípulo de Daniele, Michele Alberti, y el pintor romano Jacomo Rocchetti ${ }^{16}$, muy querido por Miguel Ángel, que según Baglione le legó algunos dibujos y cartones ${ }^{17}$, algo que corrobora el inédito inventario del pintor romano, en el que se citan más de 100 dibujos del maestro de Caprese ${ }^{18}$. Rocchetti colaboró además con Jacopo del Duca en la realización de un ciborio que, presumiblemente, era el mismo para Santa Maria degli Angeli que acabamos de mencionar ${ }^{19}$, y junto con Alberti decoraría a partir de 1572 la Capilla Cevoli, una de las primeras de la iglesia ${ }^{20}$, a la que seguirían otras en las que trabajaron Jacopo del Duca, que se encargó del sepulcro de su tío, y un antiguo seguidor de Daniele, Giulio Mazzoni, con el que el siciliano ya había trabajado ${ }^{21}$.

11 Desde 1631, San Bonaventura dei Lucchesi, en la actual Via de Lucchesi. Armellini, 1942, p. 322.

12 Romani, V., Daniele da Volterra amico di Michelangelo, Florencia, 2003. Treves, L., "Daniele da Volterra and Michelangelo: a Collaborative Relationship", Apollo, 154, 2001, 474, pp. 36-45.

${ }^{13}$ Este tabernáculo debe ser el que acordó hacer con Jacomo Rocchetti el 3 de enero de 1565. G. Redín, "Jacopo del Duca, il ciborio della certosa di Padula el il ciborio di Michelangelo per Santa Maria degli Angeli", Antologia di Belle Arti, 63-66, 2002, pp. 125-138 (135). La carta en BArocchi, P., LOACH, K., Ristori, R., Il carteggio di Michelangelo. Carteggio indiretto, 1988, Florencia, 1988, 378 y 384.

${ }^{14}$ Daelli, G., Carte Michelangiolesche inedite, Milano, 1865, p. 234. Barocchi, Loach, Ristori, 1988, 378 y 384.

15 Sobre Del Duca y su amistad con Miguel Ángel, Bernardi Salvetti, C., Santa Maria degli Angeli alle Terme e Antonio Lo Duca, Città di Castello, 1965, pp. 231-233.

${ }^{16}$ Redín, 2002, pp. 136-137.

17 Baglione, G., Le vite de' pittori scultori ed architetti dal pontificato di Gregorio XIII del 1572 in fino a' tempi di papa Urbano Ottavo, Roma, 1649, p. 66.

${ }^{18}$ El inventario de Rocchetti está adjunto a un acto en el que nombra a su mujer, Giulia, tutora de sus dos hijas, Ilaria y Eugenia, y se citan en él "disegni di mano di michelangelo bona rota numero di cento pezzi e più... disegni di mano di m. Jacomo Rocchetti pittore numero di trecento pezzi e cartoni numero di pezzi venti tra forme e modelli di cera e di gesso numero di pezzi quaranta in circa". ASR. TNT (Trenta Notai Capitolini). Uff. (Ufficio) 13, 95, 18 julio 1596 , ff. $809-810$ y $821-823$

19 REDín, 2002, p. 136.

${ }^{20}$ Masetti Zanini, G. L., Pittori della seconda metà del Cinquecento in Roma, Roma, 1976, pp. 93-95. PugLiatTI, T., Giulio Mazzoni e la decorazione a Roma nella cerchia di Daniele da Volterra, Roma, 1984, pp. 172-173.

21 Y que tenía buenas relaciones con él, pues antes de volver a su Piacenza natal, le nombró procurador para sus asuntos en Roma. Redín, 2002, p. 136. 
Vasari cuenta que Daniele quiso se colocara sobre su tumba la escultura del ángel que Paulo IV le había encargado para la entrada a Castel Sant'Angelo, legando para ello 200 escudos a sus discípulos Michele Alberti y Feliciano de San Vito para que la terminaran ${ }^{22}$, pero en realidad la tarea y el dinero fueron encomendados, en exclusiva, a Feliciano de San Vito, el "creato et alumno" que Ricciarelli debía considerar más capacitado como escultor. La estatua era un San Miguel $^{23}$, y su colocación sobre la tumba del volterrano en Santa Maria degli Angeli puede manifestar su devoción al culto angélico promovido por Antonio del Duca. Sin embargo, la voluntad de Daniele es también un signo claro de la consideración que tenía de sí como artista, y especialmente como escultor, ocupación a la que se dedicó casi exclusivamente la última década de su vida, siguiendo los pasos de Miguel Ángel, que, como es sabido, esculpió durante sus últimos años la Pietà de la Accademia de Florencia para su propia tumba. De esta manera, Ricciarelli continuaba con la actitud reivindicativa de otros artistas florentinos, como Baccio Bandinelli, con la Piedad para su sepulcro en la Santissima Annunziata de Florencia, o Benvenuto Cellini, con su Crucifijo de mármol, hoy en El Escorial. El ángel de Daniele se encontraba en la casa de Fabio de Bentornati y no, como pensaba Gasparoni, en la "stanza della figura" de la casa de Miguel Ángel alquilada por Daniele ${ }^{24}$.

La relación de Feliciano con su maestro se remonta al menos hasta abril de 1559, cuando aparece como testigo en un documento relativo al transporte desde Porto Ercole a Roma de un bloque de mármol de Carrara que Ricciarelli había escogido para la realización de la Capilla Ricci en San Pietro in Montorio, y que debía recoger Michele Alberti ${ }^{25}$. Feliciano era hijo del notario Pietro Honofri de San Vito y estaba relacionado con el entorno de Alberti, a cuya hermana Perpetua devolvió en octubre de 1576 un préstamo de cien escudos ${ }^{26}$. Siempre es citado como escultor, pero sólo hemos podido localizar una noticia referente a su trabajo. Se trata de una estatua de "un David che amazza Golia" por la que en septiembre de 1581 el pintor Cola de Amicis de Genazzano, que había sido intermediario en su venta, le devolvió 20 escudos ${ }^{27}$. Nicolaus de Amicis, alias Cola, era, al menos desde 1569, marido de Vincentia Rocchetta, y padrastro de Jacomo Rocchetti ${ }^{2}$, el socio de Michele Alberti. Feliciano compareció en una reunión de la cofradía de San Giuseppe di Terrasanta en abril de $1587^{29}$, una institución asistencial

22 VASARI, 1568, V, p. 549.

${ }^{23}$ De un San Miguel para la nueva entrada a Castel Sant'Angelo se habla en un pago de la Camera Apostólica recibido por Daniele el 3 de marzo de 1556. Bertolotti, A., Artisti lombarda a Roma nei secoli XV, XVI e XVII, Milán, 1881, pp. 133-134. PugliatTi, 1984, p. 279.

24 Sita en Macel de' Corvi, junto a la iglesia de Santa Maria de Loreto, por eso no aparece en el inventario de la casa de Daniele. En el acto relativo al alquiler, fechado el 1 de mayo de 1564, son testigos Jacopo Rocchetti y Jacopo del Duca. Daniele invirtió bastante dinero en mejorar la casa, pero Leonardo Buonarroti se reservó el derecho a usar la zona de la torre. GASPARONI, 1866, pp. 159-163.

25 Masetti Zanini, 1974, p. 92. Pugliatti, 1984, p. 279.

${ }^{26}$ A veces su nombre o apellido se cambia, como en este acto, donde es citado a la vez como "Felitiano de Nosiis de Sancto Vito" y "Felitianus q. notarii petri de honofris a castro de Sancto Vito". ASR. TNT. Uff. 20, Peracha, 7, 23 octubre 1576, f. 427. Un acto en el que Felitiano entrega la dote del matrimonio de su cuñada Silvia indica que vivía entonces cerca de San Silvestro al Quirinale, junto a la casa de Daniele. ASR. TNT. Uff. 2, Richettus, 71576,15 junio 1576, f. 270. La devolución del préstamo en ASR. TNT. Uff. 2, 7, Ricchettus, 15 junio de 1576, f. 270. En junio de 1568 recibía 60 escudos de una sociedad establecida con el "muratore" Guido di Francesco de Cicigliano. ASR. TNT. Uff. 18, Bonincontris, 6, 23 junio 1568, f. 233.

27 ASR. CNC. Lancisi, 932, 23 septiembre 1581, f. 234.

28 Vincentia era la antigua esposa de Michele Rocchetta, padre de Jacomo. ASR. TNT. Uff. 13, Vola, 34, 17 de octubre de 1569, f. 249.

${ }^{29}$ Figura en la reunión como "Felitanus Nosii de Sancto Vito". ASR. TNT. Uff. 11, Richettus, 7, 11 de abril, f. 514. El cinco de abril del mismo año recibía un pago del marido de su prima Silvia, el "scarpellino" Sebastianus de Pollis, respecto a una casa que Feliciano y él habían alquilado conjuntamente. ASR. CNC. Lancisi, 932, 5 abril 1587, f. 22. 
integrada sobre todo por $\operatorname{artistas}^{30}$, que un año más tarde colaboró en la consecución de una dote para la hija del escultor ${ }^{31}$.

Michele Alberti es el "creato et alumno" más conocido de Ricciarelli. Siempre se ha creído que formaba parte de la dinastía artística de los Alberti de San Sepolcro ${ }^{32}$, pero lo cierto es que aparece en los documentos como romano y que su padre se llamaba Alessandro y no Alberto, nombre del fundador de la rama más importante de la familia aretina ${ }^{33}$. Daniele legó a Michele 75 escudos que debían ser reclamados a los herederos de Lucrezia della Rovere, una demanda que se debe relacionar con la decoración de la capilla patrocinada por Lucrezia en la iglesia de Trinità dei Monti. La capilla fue concedida a la noble en agosto de 1548, en julio de 1550 Giovanni Paolo Rosetti, autor del fresco con la Presentación de Jesús en el templo, finiquitaba su relación con Ricciarelli, y en enero de 1553 se realizaba una estima de lo realizado hasta entonces, probablemente porque tras la muerte de la comitente en 1552 la obra se había para$\mathrm{do}^{34}$. Según Vasari, Daniele empleó nada más y nada menos que 14 años en terminar este trabajo $^{35}$, dato que algunos han considerado malintencionado por parte del aretino, que continuamente contrapone su "prestezza" y resolución en la ejecución de sus encargos a la extrema lentitud de Daniele. Pero en este caso parece estar en lo cierto, visto que 18 años después de su inicio los Della Rovere adeudaban 75 escudos a Ricciarelli ${ }^{36}$. Además, por lo que estamos viendo, el testamento demuestra que Vasari estaba muy bien informado, algo normal, si tenemos en cuenta que se carteaba con Michele Alberti ${ }^{37}$, que precisamente debía reclamar los 75 escudos a los herederos de Lucrezia. ¿Se reclamaría ese dinero por la finalización de la capilla? Siempre se ha considerado que La matanza de los inocentes que Ricciarelli regaló a la ciudad de Volterra en 1557 fue pintada después del fresco del mismo tema y casi idéntica composición de la capilla Della Rovere ${ }^{38}$. Sin embargo, el hecho de que todavía en 1566 los herederos de Lucrezia debieran a Daniele 75 escudos, una cifra importante, invita a pensar que su decoración no se había terminado mucho antes, y que Alberti pintó el fresco ya en los años 60. La tabla de Volterra es la última pintura de Daniele, que en adelante se dedicaría a la escultura, delegando la ejecución de la pintura en sus discípulos, como en el caso del Bautismo de Cristo de la Capilla Ricci en San Pietro in Montorio, que se atribuye a Alberti, y que fue contratada por Daniele en 1556, pero terminada seguramente por Alberti y Rocchetti en 1568, como

30 Tiberia, T., La compagnia di San Giuseppe di Terrasanta nel XVI secolo, 2000, p. 20.

${ }^{31}$ La ayuda para la dote era de 25 escudos, en este momento Feliciano había dejado el Quirinale y vivía en el Monte Pincio. ASR. TNT. Uff. 11, Fuschus, 10, 16 mayo 1588, f. 120. La cantidad se hizo efectiva en junio. ASR. TNT. Uff. 11, Fuschus, 10, 22 junio 1588, f. 357.

32 Matteoli, A., "Michele Alberti" en Allgemeines Kunsterlexikon, 1, 1983, ad vocem. Belli Barsali, I., Dizionario Biografico degli Italiani, I, 1960, ad vocem.

${ }^{33}$ Se le denomina Romano y escultor, e hijo de Alessandro de Albertis, en un acto referente a la compra por 100 escudos del piso de una casa en el Quirinale a cierto Carolus qd. Bernadino Stelle. ASR. TNT. Uff. 13, Vola, 67, 31 octubre 1583, ff. 536 a 539. Existió un Alessandro Alberti, pintor, hijo de Alberto Alberti, y hermano de los más famosos Cherubino y Giovanni que era de una edad similar a la de Michele Alberti, por lo que no puede ser su padre. Pugliatti pensaba, con razón, que Michele no era de Borgo San Sepolcro, porque Alberto Alberti, que cita a todos sus hijos en su diario, no menciona ninguno con ese nombre, y además Vasari fue padrino en Roma de un nieto de Alberto Alberti, por lo que, de existir un Michele en ese ramo de la familia, lo habría mencionado. PugLiatTI, 1984, pp. 71-72.

34 Pugliatti, 1984, pp. 61-68.

35 VASARI, 1568, V, p. 545.

36 REDín, 2005, p. 179, n. 693. REDín, 2007, p. 166.

${ }^{37}$ En la carta que Daniele escribe a Vasari narrándole los últimos días de Miguel Ángel, le dice que Michele le escribiría en breve. Frey II, 1930, pp. 53-58. Pugliatti, 1984, p. 171.

38 Uffizi. Inv. 1890.1429. Véase Romani, 2003, p.152. Pugliatti, 1984, p. 171. 
indica la fecha sobre el fresco del arco de entrada ${ }^{39}$. Alberti vivía en la zona del Quirinale y seguía vivo en julio de $1588^{40}$, aunque murió antes de noviembre de $1590^{41}$. Puede que durante sus últimos años, al menos desde 1583 , se dedicara también a la escultura, puesto que se le cita entonces en un documento como escultor, y no como pintor ${ }^{42}$.

Respecto a la concepción del Bautismo de Cristo de la capilla Ricci, cabe suponer que existía un dibujo para la composición realizado por Daniele, que hizo varios estudios para sus figuras ${ }^{43}$. La figura de San Juan Bautista reproduce un dibujo para uno de los jóvenes a la carrera de la Batalla de Cascina de Miguel Ánge ${ }^{44}$, Steen Hansen considera que su utilización es un homenaje a Buonarroti, bautizado en la iglesia dedicada al santo en Caprese ${ }^{45}$. A nuestro entender, es muy extraño que Daniele utilice un motivo del maestro tan antiguo, sobre todo cuando durante su último periodo éste le proporcionaba valiosos apuntes ${ }^{46}$. Esto nos hace pensar que en la ideación de la pintura de la Capilla Ricci pudieron intervenir también Alberti y Rocca, sobre todo si tenemos en cuenta que, como ya hemos dicho, este último heredó dibujos de Miguel Ángel ${ }^{47}$. Por otra parte, la figura de Cristo arrodillado muestra la misma ejecución dura y seca, con pocos matices de claroscuro, del San Jerónimo, de similares rasgos físicos, de la Crucifixión de la capilla Cevoli, obra documentada de Rocca ${ }^{48}$, por lo que la pintura del altar de la capilla Ricci pudo ser concebida en parte, por Alberti y Rocchetti.

Junto a Felitiano de San Vito y Michele Alberti, el testamento menciona a otro "creato" de Daniele, "Blasio Bettj de Cosigliano de Pistorio", al que Baglione dedica poco más de una página en sus Vite ${ }^{49}$. Biagio Betti debió nacer en Cutigliano, Pistoia, hacia 1545 y murió en Roma en $1615^{50}$. Hacia 1572-73 ingresó en los Teatinos en San Silvestro al Quirinale, dedicándose a la escultura y a la pintura dentro del convento de su orden ${ }^{51}$ y debió colaborar en la realización de

39 Últimamente Dacos atribuye la pintura, a nuestro entender de manera poco convincente, a Antonio Campelo, artista portugués que trabajó junto con Leonardo Sormani, autor de la estatua de San Pablo de la capilla Ricci, en los apartamentos del Cardenal Ricci en el Vaticano en 1556. La atribución de la capilla a los discípulos de Daniele, sin contar las esculturas de mármol, está sustentada por una cuenta transcrita en el siglo XIX de un volumen del Archivo Ricci fechado entre 1527 a 1571. Pugliatti, 1984, pp. 171-172. Di Majo, I., en Romani, 2003, pp. 128-130. SteEN Hansen, M., "Disegno and Authorship in Daniele da Volterra's Late Church Commissions", Notizie da Palazzo Albani, 36/37, 2007/08, pp. 57-78. Sobre Campelo, DAcos, N., "Antonio Campelo à Rome: Le baptême du Christ de la chapelle Ricci à San Pietro in Montorio", II Congresso Internacional de História da Arte, 2001, Coimbra, 2005, pp. 763-776.

40 Tanto él como su hermana, Perpetua, que delegan en Rocchetti una venta realizada entonces. ASR TNT. Uff. 26, Ciocius, 9, 6 julio 1588 .

${ }_{41} \mathrm{Al}$ igual que su hermana Perpetua, porque la hermana de ambos, Margarita Alberti, nombró procurador entonces a su cuñado Jacopo Rocchetti para que se encargara de todas las cuestiones relativas a la herencia de sus hermanos. ASR. CNC. 932, Lancisi, 17 noviembre 1590, f. 676.

42 Cfr. Nota 33.

43 Treves, 2001, p. 38. Steen Hansen, 2007/08, pp. 57-78.

44 De Tolnay, CH., Michelangelo.1. The Youth of Michelangelo, Princeton, 1947, p. 219.

45 STEEn Hansen, 2007/8, pp. 71-72.

46 Al respecto, Treves, 2001, pp. 36-45. Romani, 2003, pp. 128-138.

47 Cfr. Nota 18. Rocchetti copió el proyecto de la tumba de Julio II, fechado hacia 1513. Sobre el dibujo, con su nombre, Kupfertichkabinett de Berlín (Inv. KdZ 15306). De Tolnay, Ch., Corpus dei disegni di Michelangelo, I, Novara, 1975-1980, 56r., pp. 63-64.

${ }^{48}$ Cfr. Nota 17.

49 Baglione, 1649, pp. 318-319.

${ }^{50}$ Cutigliano, tal y como señalaba Milanesi, y no Carigliano, como informa Vasari. Milanesi, G., Le opere. Giorgio Vasari. Con nuove annotazioni e commenti di Gaetano Milanesi, V, Florencia, 1881, p. 69, n. 3. VASARI, 1568, V, p. 549. Baglione, 1649, pp. 318-319.

51 Baglione, 1649, p. 318. Chiarini, M., "Biagio Betti”, en Dizionario Biografico degli Italiani, ad vocem. 
la estatua ecuestre de Enrique II, puesto que Jacomo Corte, que pretendía adquirir el caballo para el duque de Saboya, habla de un tercer ayudante de Daniele miembro de los Padres Teatinos ${ }^{52}$. Betti heredó junto con Feliciano de San Vito y Alberti todo aquello que se encontraba en la casa de Ricciarelli en el "monte Quirino" para el ejercicio de la pintura y la escultura. Esta casa debe ser la que estaba junto a la iglesia de San Gerolamo di Montecavallo y la fundición de Ceci.

Vasari critica abiertamente la exasperante lentitud con la que Daniele llevaba a cabo sus obras, lo que, siempre según el aretino, tampoco hacía que éstas fueran mejores ${ }^{53}$. Consciente de ello, Ricciarelli intentó garantizar que, tras su muerte, su "bottega" terminaría todos los encargos recibidos, asegurando, en lo posible, el futuro de su nombre y su taller, porque legó a sus tres discípulos 200 escudos a condición de que no vendieran el material para la práctica artística en ningún caso, y fuera utilizado siempre en común y "sub nomine et titulo dicti domini Danielis". Y para que fuera así, legó a sus discípulos 200 escudos que estos perderían de no cumplir con lo exigido. Lo cierto es que no conocemos ningún contrato que se refiera a obras asumidas en común por Alberti, Betti y San Vito en el que haya mención a Daniele, al que tampoco se cita en las obras asumidas por la pareja Alberti y Rocchetti. Exceptuando la estatua del ángel, Daniele no afirma nada en su testamento respecto a obras imperfectas, y su deseo de que su material artístico fuera utilizado bajo su nombre, revela claramente su objetivo de pasar a la posteridad, algo que parece haber logrado, toda vez que, cuando Baglione publica sus Vite en 1643, casi un siglo después de su muerte, incluye dos biografías de Rocchetti y Betti, citándolos como discípulos de Ricciarelli y mencionando que el primero se servía descaradamente en sus obras de los dibujos del maestro ${ }^{54}$.

Como acabamos de decir, Baglione afirma que Rocchetti fue uno de los muchos "allievi" de Daniele ${ }^{55}$, cosa que, al menos en el momento de la redacción del testamento, no era cierta, aunque, desde luego, estaba muy ligado a uno de sus discípulos, Michele Alberti, que era su socio habitual, con cuya hermana, Perpetua, se había casado ${ }^{56}$. En caso de que sus discípulos vendieran y dispersaran el material artístico heredado, Ricciarelli contempló la posibilidad de destinar los 200 escudos a la dote de una hermana de Michele Alberti, pero no sabemos si fueron a parar a manos de Perpetua o Margarita, la otra hermana de Michele ${ }^{57}$. Jacomo o Jacopo Rocchetti, o Rocca, según los documentos, fue un pintor cuya mediocre obra justifica las fuertes críticas de Baglione ${ }^{58}$, pero que ocupó un lugar importante en el entorno de Miguel Ángel, que le regaló varios dibujos y cartones algo que el maestro sólo hacía con los más íntimos ${ }^{59}$. En la sentida carta que Daniele escribió a Vasari narrándole los últimos días de Buonarroti, da a entender el cariño que el maestro sentía por Rocchetti, al que menciona como "Jacopo suo"60. Este Jacopo, que se ha identificado erróneamente con Jacopo del Duca ${ }^{61}$, es Rocchetti, porque en la carta, Daniele señala que Miguel Ángel había regalado algunos dibujos de las "Nuntiate e del Christo

52 La referencia de Corte en Documenti, II, 1879, pp. 401-402.

53 VASARI 1568, V, p. 545.

54 Baglione, 1649, pp. 66-67 y 318-319.

55 Idem, p. 66.

56 Así se deduce del testamento de Perpetua. ASR. TNT. Uff. 25, 2, 5 diciembre 1587.

57 En el testamento de Perpetua no se hace mención a los 200 escudos recibidos para la dote. Cfr. Nota 51.

58 Que censura, con razón, la dureza de su pintura. Baglione, 1649, pp. 66-67. Pugliatti, 1984, pp. 167-185. Sobre el pintor véase, sobre todo, Pugliatti, 1984, pp. 172-183. Joannides atribuye convincentemente a Rocchetti un dibujo para el profeta del lado del derecho del arco de la Capilla Ricci, y rechaza la atribución al mismo de varias figuras de la bóveda de la Capilla Sixtina, que asigna a Daniele. JoAnnides, P., "Drawings by Francesco Salviati and Daniele da Volterra: Additions and Subtractions", Master Drawings, 32, 3, 1994, pp. 230-251 (244).

${ }^{59}$ Cfr. Nota 18.

${ }^{60}$ FreY, 1930, II, pp. 53-55.

${ }^{61}$ Idem. Véase la ficha relativa a esta carta de CECCHI, A., en Romani, 2003, p. 166. 
che ora all'orto" a "Jacopo suo e compagno di Michele", que no es otro que Michele Alberti, el socio y cuñado de Rocchetti, algo que corrobora el inventario de los bienes de este último, donde se cuentan más de 100 dibujos atribuidos a Miguel Ángel, una cifra desproporcionada que sugiere que entre ellos se colaran no pocos dibujos de Ricciarelli62 ${ }^{6}$. La primera mujer de Rocchetti, Perpetua Alberti, gozaba de una buena posición económica y dictó testamento en diciembre de 1587, nombrando herederos universales a sus hermanos, Michele y Margarita, y a su marido $^{63}$, con el que se dedicaba, entre otras cosas, al comercio equino ${ }^{64}$. En 1589 el pintor aparece en dos reuniones de la "Universitas pictorum" ${ }^{65}$, y para 1595 había vuelto a casarse, muriendo un año después ${ }^{66}$.

Rocchetti, que en enero de 1565 se había comprometido con Jacopo del Duca a realizar un tabernáculo de grandes dimensiones siguiendo dibujos de Miguel Ángel, conocía a Del Duca al menos desde $1563^{67}$, y seguramente desde antes, visto que los dos gravitaban en torno a Buonarroti. La relación de Jacopo del Duca con la "cerchia" de Daniele no parece, sin embargo, que fuera muy buena. Leonardo Buonarroti había encomendado a Daniele realizar dos cabezas en bronce de su tío, pero su fundición se retrasaba porque Ricciarelli quería hacerlas a la vez que el caballo para el monumento de Enrique $\mathrm{II}^{68}$. Pocos días después de la muerte del volterrano, Jacopo del Duca respondía por carta el 18 de abril a Leonardo Buonarroti, que le preguntaba por los dos retratos en bronce de Miguel Ángel. Del Duca le contestó que ya estaban fundidos, pero que estaban "in modo che ormai se hanno da fare di nuevo con ciselli e lime; si che non so se saranno a proposito per V.S.: fate voi. Io per me vorrei avesti il ritratto della memoria di missere, non d'un altro. So quel che dico, dico per amor che vi porto, e forse, essendo vivo Daniello, l'arebbe fatta condurre a un modo che questi suoi genti non so che faranno" 69 . Es obvio que Del Duca no se fiaba lo más mínimo de la valía como escultores de los discípulos de Daniele, a los que despreciaba hasta el punto de considerar que si éstos terminaran los retratos de Miguel Ángel, Leonardo acabaría teniendo una efigie de otra persona, y no la de su tío. Probablemente Del Duca aspiraba a completar los retratos, e incluso tal vez la estatua ecuestre de Enrique II, que se ofrecieron a finalizar los discípulos de Daniele ${ }^{70}$, quienes realizaron también la figura del jinete, tal y como informa Jacomo Corte en $1583^{71}$.

${ }^{62}$ Cfr. Nota 18. Baglione señala que Rocchetti poseía dibujos de Miguel Ángel y de Daniele. Baglione, 1649, p. 66.

${ }^{63}$ Cfr. Nota 56. Rocchetti vivía en Via Magnanapoli, donde subalquiló una habitación en 1580. ASR. TNT. Uff 13, Vola, 64, 14 octubre 1580, f. 507.

${ }^{64}$ Muchos documentos refieren la actividad de Perpetua y Jacopo como compradores y vendedores de asnos y caballos, iniciada al menos en 1578, cuando Perpetua vendió varios asnos en un acto en el que figura como testigo el pintor romano Guglielmo del Frasinello. ASR. CNC. Lancisi, 932, 13 noviembre 1578, f. 35. Entre 1583 y 1585 continúa la venta de equinos. ASR. CNC. Lancisi, 932, 11 marzo 1583, 4 diciembre 1584, 27 febrero 1585 y 4 septiembre 1585 , respectivamente, ff. $292,415,428$ y 452 . En este volumen hay otras noticias, relativas a préstamos y procuras, de nulo interés artístico.

${ }^{65}$ Reuniones del 5 de enero y 22 de julio de 1589 de la Universitas Pictorum. ASR. TNT. Uff. 12, Saravezzi, 12 , 5 enero 1589, f. 123. ASR. TNT. Uff. 13, Saravezzi, 13, 22 julio 1589, f. 505.

${ }^{66}$ Con cierta Julia Faeteram de Rocchettis, a la que nombró procuradora en marzo de 1595. ASC. TNT. Uff. 13, Vola, 92, 16 marzo 1595, f. 488. En febrero de 1596 seguía vivo, cumpliendo algunas disposiciones de la herencia de Perpetua. ASC. TNT. Uff. 13, Vola, 94, 1 febrero 1596, f. 266. En abril de 1597 la hija y los herederos de Rocchetti pagaban el resto de un censo a las hijas y herederos de Marco Antonio de Bonincontris. ASC. TNT. Uff. 13, 97, 17 abril 1597.

${ }^{67}$ En agosto de 1563 Rocchetti aparece como testigo de un acto en el que Jacopo del Duca vende un censo sobre una casa. ASR. TNT. Uff. 13, Vola, 8, 20 agosto 1563, f. 186.

68 CeCCHI en Romani, 2003, pp. 170-174.

${ }^{69}$ DaelLi, 1865 , pp. $72-73$, n. 41

70 VASARI, 1568, V, p. 549.

71 BostrÖM, 1995, p. 817. 
En la correspondencia mantenida entre Simone Guiducci y Roberto Strozzi respecto a la ardua realización del caballo para la estatua de Enrique II, el primero se lamenta de la extrema lentitud del trabajo de Daniele, que después de varios meses sin dejar de pedir dinero continuamente, no había hecho todavía nada visible, por lo que debía "essere di natura agiato come li altrj di quella professione", o sea, que vivía cómodamente ${ }^{72}$. A juzgar por lo señalado en su testamento e inventario, Daniele vivía de manera holgada. Además de poseer una casa en el Quirinale, invirtió 326 escudos en el alquiler y reforma de la casa de Miguel Ángel, por la que pagaba a Leonardo Buonarroti 35 escudos anuales a condición de que éstos fueran empleados en su reforma ${ }^{73}$. Sin embargo, parece que nunca llegó a vivir en ella, pues sus cosas y su taller se encontraban en la casa de Montecavallo, tal y como refleja el inventario. Algo lógico, pues a su lado se encontraba la fundición donde se realizaba el caballo. En cualquier caso, Daniele tenía algunas pertenencias en la antigua vivienda de Miguel Ángel, porque Jacopo del Duca se queja en una carta escrita a Leonardo Buonarroti, que se reservaba la torre del edificio para sus estancias en Roma, de que el procurador no quería darle las llaves porque todavía no se había hecho la división entre sus cosas y las del recién fallecido Ricciarelli ${ }^{74}$.

Si comparamos el patrimonio de Daniele con el de Francesco Salviati, el otro gran pintor activo en Roma por entonces, fallecido en noviembre de 1563, podemos hacernos una idea de su buena situación económica. Salviati no dictó testamento, pero por lo que cuenta su amigo Vasari, había perdido "tutti gl'ufficii che aveva dopo intolerabili fatiche comperato" ", dejando a su discípulo Annibale, hijo del arquitecto Nanni di Baccio Bigio, 14 pinturas y todos los útiles para pintar, además de un título de 60 escudos al año del Monte delle farine ${ }^{76}$, mientras que a su hermana le cedió un valioso cuadro y el resto de las cosas, que al parecer fueron muy pocas, puesto que Vasari afirma que "ella non ebbe, come si dice, del sacco le corde" 77 . Sin embargo, si comparamos a Ricciarelli con su discípulo español, Gaspar Becerra, su colaborador en la Capilla della Rovere, vemos que éste, fallecido en 1568, vivió mejor que su maestro. Becerra donó en su testamento un total de 565 escudos, cantidad elevada que responde al artista mejor pagado de la corte de Felipe II, con 600 escudos anuales ${ }^{78}$. Así, Becerra no tuvo problema en devolver a su mujer, Paula Becerra, antigua "cortigiana"79, los 1.100 ducados que ésta le había dado como dote $^{80}$. Por otro lado, los 200 escudos que Daniele había contemplado que se emplearan como dote para la hermana de Michele Alberti en caso de que sus discípulos vendieran sus enseres artísticos, eran una cantidad media, que contrasta, desde luego, a pesar de los cambios del valor monetario, con los 800 escudos que Perin del Vaga dio veinte años antes como dote a su hija Lavinia Bonaccorsi, casada con el médico de Margarita de Austria, Giuseppe Cinci ${ }^{81}$. De todas

72 Idem, pp. 1810 y 1819.

73 Gasparoni 1865, p. 182. El Cardenal Bonelli la compró en 1593 y la alquiló a Scipione Pulzone. Redín, G., "Due testamenti e altri documenti su Scipione Pulzone", Ricerche di Storia dell'Arte, 78, 2002, pp. 77-86.

${ }^{74}$ Carteggio Indiretto, 1988-1995, II, pp. 249-250, n. 394. GaSParoni, 1865, pp. 158-159.

75 VASARI, 1568, V, p. 532.

76 Título de deuda pública que ofrecía un interés entre el 5 y 10\%. Delumeau, J., Vie économique et sociale de Rome dans la seconde moitié du XVIe siècle, París, 1957, pp. 791-817.

77 VASARI, 1568, V, p. 532.

78 Llaguno, E., Noticias de los arquitectos y arquitectura de España desde su restauración, II, Madrid, 1977 (1829), pp. 259-260. Barbeito, J. M., El Alcázar de Madrid, Madrid, 1992, p. 43.

${ }^{79}$ ReDín, 2007, pp. 160 y 190-192.

${ }^{80}$ Y además compró con ella una capilla en el Convento de la Victoria de Madrid. Arias Martínez, A., "Gaspar Becerra escultor o tracista. La documentación testamentaria de su viuda, Paula Velázquez", A.E.A., 283, 1998, pp. 273288. Herrera García, F. J., "Gaspar Becerra: su entorno familiar y profesional a la luz de nuevas fuentes documentales", Boletín del Museo e Instituto Camón Aznar, 93, 2004, pp. 59-95.

${ }^{81}$ Que Cinci recibió dos años más tarde. REDín, 2007, p. 241. 
maneras, Jacomo Corte calculaba en 1583 que de los 6.000 escudos que había costado el caballo de Enrique II, Daniele se habría llevado por su trabajo 2.000, una cantidad a la que no se hace referencia alguna en el testamento ni en el inventario, y que no sabemos si resulta exagerada o bien si el artista hubo de afrontar por su cuenta gastos inesperados, ligados seguramente a la realización del bronce ${ }^{82}$.

El inventario de los bienes de Daniele se hizo a petición de los Rucellai, que habían sostenido la financiación de la estatua ecuestre y que en 1586 se llevaron el caballo a su palacio romano ${ }^{83}$. El inventario Corvisieri parece el mismo, transcrito parcialmente sin indicar su ubicación, publicado por Gasparoni, y fue realizado los días 5 y 6 de abril de $1566^{84}$. Lo presenciaron Michele Alberti y Feliciano de San Vito (Betti estaba enfermo), que, en posesión de los bienes, se comprometieron a entregarlos a quien designara el juez de la "Camera Apostolica". Gasparoni se ocupó fundamentalmente de las obras de arte cuyo tema es indicado ${ }^{85}$, y el inventario no añade a este respecto ninguna novedad, pero nos permite conocer el ajuar del artista y saber algo sobre cómo vivía. Seguramente Daniele compartía casa con alguno de sus ayudantes, porque en ella había vajilla suficiente para varias personas, tres camas, cinco colchones, una silla para dormir y una "Cariola con materasso", o sea, un carrito con colchón para niños que debían usar los hijos de alguno de sus colaboradores. A juzgar por la calidad y la cantidad de sus ropas, no hay duda de que Daniele vivía con holgura. Todo su vestuario era negro, como el del propio Miguel Ángel, un color que, según Castiglione, correspondía a los caballeros ${ }^{86}$, algo que tal vez pueda relacionarse con la espada citada en la habitación de Ricciarelli. Entre sus ropas se cuentan dos pellizas y tres prendas de "rascia", la tela de lana más cara fabricada en Florencia, una de las cuales estaba forrada en piel, algo que, junto con los dos caballos que poseía, suponía un lujo inusual para un artista del Renacimiento ${ }^{87}$, y que habla a las claras de su situación económica y social, recordando las palabras de Vasari a Jacone, que otrora se reía del joven aretino, sobre su bienestar "Io ero già povero come tutti voi et ora mi trovo tre mila scudi o meglio (...) io già serviva voi altri, et ora questo famiglio che è qui serve me e governa questo cavallo; vestiva di panni che vestono i dipintori che son poveri, et ora son vestito di velluto; andava già a piedi et ora vo a cavallo"88.

En el inventario publicado por Gasparoni se mencionan, entre cabezas y bustos, seis retratos de Miguel Ángel en bronce, mientras que en el que se encuentra en la Miscellanea Corvisieri del Archivio di Stato di Roma se cuentan sólo cinco $^{89}$, faltando una de las cabezas situadas en la "stanza da basso". Por lo que respecta a su autoría, es probable que alguna de ellas hubiera sido

82 Por su parte Filippo Bucci, agente de Corte, afirma que Daniele recibió 1700 escudos y que los asistentes que pulieron el caballo recibieron 400. BoströM, 1995, p. 818. Documenti inediti, II, p. 403.

${ }^{83}$ Idem, p. 809.

${ }^{84}$ Y no sólo el 5 de abril, como indicaba Gasparoni, que tampoco dice nada acerca de la presencia de los discípulos de Daniele. GASPARONI, 1865, pp. 178-180.

${ }^{85}$ Gasparoni, 1865, pp. 170-180, nn. 6-12. Respecto al cartón de la Leda citado en el inventario, Joannides cree, muy razonablemente, que se puede relacionar con la escultura de Dido conservada en Berlín que deriva del perdido cuadro de Dido y Eneas de Daniele. JoAnnides, P., "Daniele da Volterra's Dido", The Burlington Magazine, 135, 1993, pp. 818-819. Del resto de los cartones citados no se tiene noticia, y Gasparoni no argumentó sus posibles identificaciones, como tampoco la del cartón de la Pietà de mano de Miguel Ángel que, sin razón alguna, consideró el de la "Madonna della febbre". Por lo que respecta al retrato de Miguel Ángel sobre tabla probablemente sería obra de Daniele, que lo había retratado ya en la Capilla della Rovere.

86 De Castiglione, B., Il libro del Cortegiano, II, Venecia, 1528 (ed. Preti, G., Roma, 1965), p. 130.

87 Sobre lo excepcional de poseer un caballo y la rica vestimenta, también de "rascia", en el caso de Miguel Ángel, Wallace, W. W., "Miscellanea curiositae Michelangelae: a Steep Tariff, a Half Dozen Horses, and Yards of Taffeta", Renaissance Quarterly, 47, 1994, pp. 330-350 (336-339).

88 VASARI, 1568, V, p. 405.

89 CORBO, 1965, p. 107. 
terminada por Michele Alberti, que, al fin y al cabo, era también escultor ${ }^{90}$, y que escribió el 18 de abril de 1566 a Leonardo Buonarroti diciéndole que los bronces se repulirían pronto y que en un mes los recibiría ${ }^{91}$. Se ha pensado que los dos retratos comisionados por Leonardo se hicieron a partir de una máscara mortuoria ${ }^{92}$, pero lo cierto es que en el libro de gastos del sobrino de Miguel Ángel se menciona la compra de cera trementina "per fare gittare due teste di Mich(elagno) Buonarroti, per fare le cere per la forma"93. La "forma", esto es, el molde; no se dice nada respecto a una máscara. Además, el marcado gesto entre melancólico y sufriente de los rostros de estos bronces no tiene nada que ver con la laxitud propia de las facciones de una máscara mortuoria. Como ha señalado Di Majo, su modelo fue el cartón para el rostro de Miguel Ángel de la Asunción de la Capilla Della Rovere ${ }^{44}$ y así lo demuestra la correlación de todas las arrugas y marcas de expresión, e incluso el recorrido de los rizos sobre la frente del maestro, del que, por lo demás, había en casa de Daniele un retrato pintado sobre tabla.

Gasparoni centró su atención en la habitación de Daniele, donde se encontraban casi todos sus dibujos y libros. De los últimos sólo se identifican un Vitruvio, que demuestra el lógico interés de Daniele por la arquitectura, corroborado por "un libro d'architettura scritto a mano", que Gasparoni no menciona; un Plinio, quizá su Naturalis historiae; un Dante, tal vez la Divina comedia; un libro "intitolato alberto duro in stampa", que tal vez sea alguno de los tratados de Durero; y una de sus dos Pasiones, que seguramente estaba acompañada de la otra, puesto que en el inventario se habla inmediatamente de otro libro "simile" 95 . Se trata de volúmenes comunes en la biblioteca de cualquier artista, pero la probable presencia de un tratado de Durero no deja de resultar chocante, habida cuenta de las críticas de Miguel Ángel hacia el método proporcional del alemán ${ }^{96}$. El último libro citado — pero había más en un escritorio lleno de libros y dibujos- es un librito de "Ricordi" de Daniele en el que se encontrarían anotados todos sus gastos y la actividad económica referente a sus trabajos.

Entre la muchísima obra gráfica citada, dividida según contenido en libros de retratos, grutescos, "disegni di cappella" y "schizzi et inventioni" "97, llaman la atención diecisiete cabezas al óleo sobre papel, que probablemente eran estudios finales para las composiciones una vez terminadas, como el cartón del rostro de Miguel Ángel para la Asunción Della Rovere conservado en el Teylers Musem. Sabido es que Daniele modelaba esculturas en cera para crear sus figuras ${ }^{98}$, haciendo incluso, tridimensionales, las invenciones sobre el papel de otros autores, como Rafael, en la Matanza de los inocentes pintada para Volterra ${ }^{99}$. Por eso, las treinta cabezas de la habita-

90 Cfr. Nota 33.

91 DAELLI, 1865, pp. 72-73.

92 CeCchi en Romani, 2003, pp. 170-172.

93 Idem, p. 170. Carteggio indiretto, 1988-1995, II, p. 199, n. 9.

94 Di MAJO en Romani, 2003, pp. 110-111.

95 Inevitable pensar en Miguel Ángel, estudioso de Dante, cuya obra, según Condivi, conocía de memoria. CoNDIVI, A., Vita di Michelagnolo Buonarroti raccolta per Ascanio Condivi, Roma, 1553, p. 45.

${ }^{96}$ Libros similares poseía Gaspar Becerra, algunos heredados por su mujer, Paula como "un libro de Vitruvio en toscano", "un libro de Alverto de Juane", quizá alguna de las dos Pasiones de Durero o, como sugiere Herrera, la Simetría y la Geometría de Durero, lo que indica que la asimilación del arte de Buonarroti no fue tan ciega ni tan dogmática como en alguna ocasión se ha pensado. Herrera 2004, p. 83. Redín, 2007, p. 228. CONDIVI, 1553, p. 42.

97 GASPARONI olvida el libro "di disegni di capella legato in corame pagonazzo" citado en el inventario.

98 Yesos aparte, en su habitación se menciona un escritorio lleno de figuras de cera. De la pintura de David y Goliat, hoy en el Louvre, que en 1715 fue regalada por el Príncipe di Cellamare, Domenico del Giudice, embajador de España en Francia, a Luis XIV como obra de Miguel Ángel, existe un grabado realizado con tal motivo por Benoît Audran fechado entonces. Su existencia me ha sido amablemente señalada por Gabriele Finaldi. Gallerie Cabotse. Catalogue d'estampes. 1. abril 2009, p. 33.

99 Como ha demostrado brillantemente Romani, 2003, p. 152. 
ción de los yesos podrían ser consideradas estudios tridimensionales para las propias pinturas, relacionadas con las cabezas al óleo, y no retratos o modelos para dibujar (¿para qué tantas cabezas?), porque, que sepamos, la producción escultórica de Daniele fue más bien escasa. En la misma medida habría que considerar las "infinite teste" guardadas en la habitación más alta de la casa, y más si pensamos en la extraordinaria cercanía existente entre el cartón del rostro de Miguel Ángel y su plasmación en escultura, que invita a pensar que Daniele pudo modelar el rostro del maestro antes o al mismo tiempo que lo dibujaba. De esta manera, al tomar como punto de partida para las figuras de sus cuadros una escultura, sea una cabeza, un torso, brazo o pierna, Ricciarelli se garantizaba el efecto de relieve escultórico al que aspiraba. En la casa de Daniele había una habitación llamada "Stanza di San Pietro" porque en ella estaba una estatua del santo en estuco que debía ser el modelo de la escultura de la Capilla Ricci ${ }^{100}$. La otra escultura de la capilla, un San Pablo, se atribuye a Leonardo Sormani ${ }^{101}$. La ausencia de una escultura en estuco dedicada a este santo en casa de Ricciarelli puede sugerir que no modeló ninguna para la capilla Ricci, limitándose a concebirla, aunque puede ser que no estuviera en su taller precisamente porque fue trasladada al de Sormani ${ }^{102}$.

Daniele nombró heredero universal a su tío Leonardo Ricciarelli, al que cedió todos sus bienes muebles e inmuebles, que, sin embargo, no son referidos en el testamento ni especificados en el inventario. Como ejecutor del testamento se cita a Jo. Franciscum Letterium, canónigo de la basílica de San Pedro, cuyo arbitrio debían asumir los herederos del artista en caso de disputa en la repartición de bienes. La distribución de la herencia no fue, de hecho, sencilla: el 8 de junio de 1566, Leonardo Ricciarelli, por un lado, y Michele Alberti, Feliciano di San Vito y Biagio Betti, por otro, decidieron terminar sus disputas respecto al legado de Daniele, para lo que acordaron aceptar la decisión que sería tomada por los jueces Lentullus Cappellus y Petrus Paulus Piattetti, pagando, de lo contrario, una multa de 200 escudos de oro; si las dos partes no estuvieran de acuerdo con dicha decisión, se esperaría a conocer la opinión de un tercer árbitro ${ }^{103}$. Pocos días más tarde, el 20 del mismo mes, los denominados "discreti iuvenes" Michele Alberti, Feliciano de San Vito y Biagio Betti, de común acuerdo, anularon el depósito de los bienes realizado a petición suya por Augustini de Pellegrini (seguramente el comense citado entre los testigos del testamento de Daniele), dando potestad a Leonardo Ricciarelli para que se ocupara de sus intereses sobre la herencia de Daniele e intentara recuperar parte de la misma ${ }^{104}$. Probablemente, se referían a la recuperación del dinero que su maestro había gastado en la reforma de la antigua casa de Miguel Ángel que Leonardo Buonarroti le había alquilado, porque el 21 de mayo de 1567 se reclamaba al último, dueño de la casa, el pago de esas mejoras, que suponían en total 259 escudos de moneda ${ }^{105}$, que no se saldaron hasta noviembre de $1574^{106}$.

El testamento resulta escaso en informaciones directas sobre la actividad de Daniele, que queda reflejada en su inventario, donde, por cantidad, se impone todo el material empleado en la realización del caballo de Enrique II y, en general, en la práctica de la escultura ${ }^{107}$. A diferencia

100 GASPARONI, 1865, p. 180.

101 Pugliatti, 1984, pp. 179-182. Steen Hansen, pp. 64-66.

102 En la casa de Daniele había, además, un "cavo della testa di san pietro", o sea, un molde de su cabeza.

103 ASR. TNT. Uff. 13, Vola, 39, 8 junio 1566, f. 136 v.-138 r.

104 ASR. TNT. Uff. 13, Vola, 39, 20 junio 1566, f. 155 r.-156 r.

105 Restando dos meses de alquiler, a 35 escudos cada uno. GASPARONI, 1865, p. 176.

106 Carteggio indiretto, 1988-1995, pp. 292-293, n. 423.

107 Gasparoni pasó por alto en la "Stanza da basso" un caballo de metal, seguramente relacionado con el caballo de Enrique II, además de 33 medallas en la habitación de Daniele, junto a varias hojas de pan de oro, una cajita con sus pinceles y otra de colores con "lacca di grana", la más cara de todas. Por lo demás, cabe mencionar entre los útiles del pintor la "sedia da dipingere" y el "ponte da dipingere". 
de sus contemporáneos, Riciarelli no se sentía muy ligado a ninguna cofradía romana, ni siquiera a la de San Luca, que le correspondía como pintor: sólo aparece en seis reuniones del amplio periodo del que se conservan registros de la institución, y en general la frecuentaba sólo durante la reunión anual en la que se elegían los cargos de la compañía ${ }^{108}$. Tampoco aparece en su testamento donación alguna a la cofradía de San Giuseppe di Terrasanta, formada por muchos artistas $\mathrm{y}$, por otra parte, llama la atención que, a diferencia de muchos de sus contemporáneos -y pensamos en los pintores españoles activos por entonces en Roma, Pietro Pisa o Domenico Treceño ${ }^{109}$, Daniele no quiso invertir en ninguno de los "monti" romanos, o al menos no se recoge nada al respecto en su testamento ni en su inventario. Más chocante resulta todavía que no donara ni un solo escudo a alguno de los hospitales para hombres, mujeres, pobres y peregrinos de Roma, o a otras asociaciones caritativas, siempre presentes en los testamentos romanos. El único donativo de Daniele sería la estatua de San Miguel legada a Santa Maria degli Angeli, un regalo, al fin y al cabo, un tanto interesado, puesto que la escultura, de la que nada se sabe ${ }^{110}$, debía ser colocada precisamente sobre su tumba.

\section{ASR, COLLEGIO NOTAI CAPITOLINI, 1772. Notario Thomassinus, f. 284 r y v.}

“Testamentum domini Danielis Ricciarelli de Vulterra die 4 aprilis 1566.

In Dei nomine amen. Anno Domini 1566 Indictione nona tempore pontificatus sanctissimi in Christo patris et domini nostri domini Pii divina providentia papae quinti, sui pontificatus anno primo, die vero 4 mensis aprilis dicti anni.

In presentia mei notarii et testium infrascriptorum ad haec specialiter habitorum, vocatorum et rogatorum presens et personaliter constitutus magnificus et excellens dominus Daniel Ricciarellus de Vulterra incola Urbis, infirmus corpore tamen Dei gratia et bonitate mente et intellectu sanus, et ut prudens volens providere si mori contigerit ex presenti aegritudine, quod tamen Deus avertat, nec post eius mortem de bonis suis aliqua lis oriatur.

Ideo hoc nuncupativum, quod sine scriptis dicitur, facere curavit et fecit testamentum in hunc qui sequitur modus et forma.

Et in primis et ante omnia humilliter et devote commendavit animam suam omnipotenti Deo eiusque gloriosae matri semper virgini Mariae et omnibus sanctis celestis patriae, et si mori contigerit ex presente aegritudine voluit et mandavit eius corpus sepelliri in ecclesia Sanctae Mariae de Angelis ad Terminos, cui reliquit ius sepulturae, et similiter voluit et mandavit quod eius corpus associetur cum sacerdotibus et funeralibus arbitrio propter absentiam sui heredis infranominandi infrascripti executori et legatariorum.

Item ipse testator voluit et mandavit quod dentur et assignentur Felitiano de Caninio de Sancto Iusto suo creato scuta ducenta monetae ut dictus Felitianus possit perficere quandam figuram angeli nunc existentem inperfectam in domo Fabii Bentornati et illa sic finita apponatur supra sepulturam in dicta ecclesia Sanctae Mariae de angelis ipsius testatoris.

${ }^{108}$ Leproux, G. M., "La corporation romaine des peintres et autres de 1548 a 1574", Bibliothèque de l'École des chartes, París, 149, 1991, pp. 293-349.

109 Redín, 2007, pp. 262-265 y 273-277. Más comerciantes que pintores, como el flamenco Gaspare Rem, que dictó testamento en Venecia en 1607, legando a su familia un patrimonio de 1200 ducados que, como señala Borean, supone una condición económica inusual para un artista de segundo orden, cuyos beneficios llegaron en su caso por la venta de pintura en los circuitos internacionales. BOREAN, L., "Inventari e testamenti d'artista nel Cinquecento", en V. V. A. A., Il collezionismo d'arte a Venezia: dalle origini al Cinquecento, Venezia, 2008, pp. 122-131.

110 Aunque Vasari dice que sus discípulos cumplieron su voluntad "con amore e diligenza", la estatua no es mencionada en la historia de la basílica que escribió en 1611 Matteo Cattalani, sucesor de Antonio del Duca como prior de la misma. Cattalani, M., Historia dell'erettione della chiesa di S. Maria degli Angeli, 1611. Cod. Vat. Lat. 8735, Biblioteca Vaticana. Salvetti, p. 103. REDín, p. 137, n. 28. 
Item iure legati reliquit Micchaeli de Albertis suo creato et alumno scuta septuaginta quinque exigenda ab heredibus Illustrissimae dominae Lucretiae de Ruvere Columnae.

Item similiter iure legati legavit et reliquit ac etiam in remuneratione laborum eidem testatori prestitorum dictis Micchaeli, Felitiano et Blasio Bettj de Cosigliano de Pistorio, suis creatis et discipulis omnia massaritia et bona in sua domo existentia ad usum exercitii tam sculturae quam picturae facienda et alia quaecumque bona sua inibi existentia.

Item similiter iure legati legavit et reliquit supradictis Micchaeli, Felitiano et Blasio scuta ducenta monetae pro quolibet quae bona capi possint propria autem ipsorum legatariorum facto tamen de eis inventario et quae semper sint existere debeant in comune ad exercitium sculturae et picturae sub nomine et titulo dicti domini Danielis testatoris cum prohibitione expressa quod non possint alienari tam bona predicta ad exercitium sculturae et picturae quam dicta scuta ducenta ut supra cuilibet relicta que sint pro capitali nomine in perpetuo dicti Danielis, reservato tamen eidem Michaeli quod si voluerit maritare suam sororem tunc et eo casu possit dicta scuta ducenta habere in casum dotis et non alias.

Cum pacto tamen et condictione expressa quod dicti Micchael, Blasius et Felitianus debeant in amorem et charitatem semper in exercitio praedicto manere simul ad effectum in dicta professione efficiant excellentes, et si defecerint unus vel plures ex eis eligatur alius vel alii de exercitio, arbitrio superviventium.

Et si superviventes essent in electione discendere tam in electione dicti deficentis quam in alium super exercitio et societate predicta inter dictos legatarios et alios postea nominandos.

Tunc debeant stari super premissis arbitrio R. D. Io. Francisci Lettitii canonici sancti Petri de Urbe, cui judicio et sententia debeant stari nec desuper per quamcumque reclamari sub pena caduci legatororum predictorum illius respective qui contravenerit.

Cum condictione et pacto expresso quod iidem Micchael, Felitianus et Blasius supranominati non possint aliquid pro eorum respective servitiis eidem testatori relevare petere a suo infrascripto herede sed contentus sive supra dictis legatis.

In quibus eosdem pro equali portione, cum pactis in supra appositis heredes instituit in omnibus dicti testatoris bonis mobilibus et inmobilibus nominibus et actionibus quibuscumque ubique existentibus instituit deputavit creavit et ore proprio nominavit suum universalem heredem D. Leonardum Ricciarellum de Vulterra suum patruum abserens ipse d. Daniel testator hoc esse suum ultimum testamentum et suam ultimam voluntatem quam et quod prevalere voluit premissis aliis testamentis et omnibus aliis ultimis voluntatibus per eum hactenus factis et si iure testamenti non valeret valere voluit iure codicillorum et si iure codicillorum non valeret valeat et valebit iure donationis inter vivos et si iure donationis inter vivos non valeret valeat et valebit iure donationis causa mortis non valeret valeat et valebit omni alio meliori iure iure forma quibus magis et melius ut suum sortiatur effectus cassans irritans et annullans omne aliud testamentum et omnem aliam ultimam voluntatem per eum hactenus factas.

Executorem autem huius testamenti et ultimae voluntatis fecit et nominavit supradictum d. Jo. Franciscum Letterium ibidem presentem et acceptantem super quibus omnibus et singulis ut premittitur sic factis et gestis rogavit notarium infrascriptum ut unum vel plura conficerem instrumentum et instrumenta nonnulla veritatis.

Actum hoc Romae in regione Montium in domo domini Attilii Cecii iuxta sua latera quem ad presens ipse d. testator inhabitabat coram et presentibus his testibus videlicet R. P. domino Jo: Angelo de Bonettis de Bressia priore monasterii sancti Hieronymi de monte Cavallo, d. Augustino de Pellegrinis laico comensi, Sancto q. Antonii de Baldo perusino, Io. Domenico q. Gregorii de Camoro comitatus spoletano, Antonio Dominici de Antoniis florentino, Laurentio del Moro de Pelicia de Como et Petro Laurentii Pelliccioni de Colle laicis testibus ad praemissa habitis etc."

Fecha de recepción: 5-XI-2009

Fecha de aceptación: 15-I-2010 